\title{
THE SECOND PINCHING THEOREM FOR HYPERSURFACES WITH CONSTANT MEAN CURVATURE IN A SPHERE*
}

\author{
HONG-WEI XU AND ZHI-YUAN XU
}

\begin{abstract}
We generalize the second pinching theorem for minimal hypersurfaces in a sphere due to Peng-Terng, Wei-Xu, Zhang, and Ding-Xin to the case of hypersurfaces with small constant mean curvature. Let $M^{n}$ be a compact hypersurface with constant mean curvature $H$ in $\mathbb{S}^{n+1}$. Denote by $S$ the squared norm of the second fundamental form of $M$. We prove that there exist two positive constants $\gamma(n)$ and $\delta(n)$ depending only on $n$ such that if $|H| \leq \gamma(n)$ and $\beta(n, H) \leq S \leq \beta(n, H)+\delta(n)$, then $S \equiv \beta(n, H)$ and $M$ is one of the following cases: (i) $\mathbb{S}^{k}\left(\sqrt{\frac{k}{n}}\right) \times \mathbb{S}^{n-k}\left(\sqrt{\frac{n-k}{n}}\right), 1 \leq k \leq n-1$; (ii) $\mathbb{S}^{1}\left(\frac{1}{\sqrt{1+\mu^{2}}}\right) \times$ $\mathbb{S}^{n-1}\left(\frac{\mu}{\sqrt{1+\mu^{2}}}\right)$. Here $\beta(n, H)=n+\frac{n^{3}}{2(n-1)} H^{2}+\frac{n(n-2)}{2(n-1)} \sqrt{n^{2} H^{4}+4(n-1) H^{2}}$ and $\mu=$ $\frac{n|H|+\sqrt{n^{2} H^{2}+4(n-1)}}{2}$.
\end{abstract}

\section{Introduction}

Let $M^{n}$ be an $n$-dimensional compact hypersurface with constant mean curvature $H$ in an $(n+1)$-dimensional unit sphere $\mathbb{S}^{n+1}$. Denote by $S$ the squared length of the second fundamental form of $M$ and $R$ its scalar curvature. Then $R=n(n-1)+n^{2} H^{2}-S$. When $H=0$, the famous pinching theorem due to Simons, Lawson, and Chern, do Carmo and Kobayashi (2], 9], [13]) says that if $S \leq n$, then $S \equiv 0$ or $S \equiv n$, i.e., $M$ must be the great sphere $\mathbb{S}^{n}$ or the Clifford torus $\mathbb{S}^{k}\left(\sqrt{\frac{k}{n}}\right) \times \mathbb{S}^{n-k}\left(\sqrt{\frac{n-k}{n}}\right), 1 \leq k \leq n-1$. Further discussions have been carried out by many other authors (see [7], 10], 14], 17], 18], 23], etc.). In 1970's, Chern proposed the following conjectures.

Chern Conjecture I. Let $M$ be a compact minimal hypersurface with constant scalar curvature in $\mathbb{S}^{n+1}$. Then the possible values form a discrete set. In particular, if $n \leq S \leq$ $2 n$, then $S=n$, or $S=2 n$.

Chern Conjecture II. Let $M$ be a compact minimal hypersurface in $\mathbb{S}^{n+1}$. If $n \leq S \leq 2 n$, then $S \equiv n$, or $S \equiv 2 n$.

In 1983, Peng and Terng made breakthrough on the Chern conjectures I and II. They 11] proved that if $M$ is a compact minimal hypersurface with constant scalar curvature in

*2000 Mathematics Subject Classification. 53C24; 53C40.

Keywords: Hypersurfaces with constant mean curvature, Rigidity, Scalar curvature, Clifford torus.

Research supported by the Chinese NSF, Grant No. 11071211, 10771187; the Trans-Century Training Programme Foundation for Talents by the Ministry of Education of China. 
the unit sphere $\mathbb{S}^{n+1}$, and if $n \leq S \leq n+\frac{1}{12 n}$, then $S=n$. Moreover, Peng and Terng [12] proved that if $M$ is a compact minimal hypersurface in the unit sphere $\mathbb{S}^{n+1}$, and if $n \leq 5$ and $n \leq S \leq n+\tau_{1}(n)$, where $\tau_{1}(n)$ is a positive constant depending only on $n$, then $S \equiv n$. During the past two decades, there have been some important progress on these aspects(see [1], 4], 5], 8], 15], [16], 24], etc.). In 1993, Chang [1] solved Chern Conjecture I for the case of dimension 3. In [4] and [5], Cheng, Ishikawa and Yang obtained some interesting results on the Chern conjectures.

In 2007, Suh-Yang and Wei-Xu made some progress on Chern Conjectures, respectively. Suh and Yang [15] proved that if $M$ is a compact minimal hypersurface with constant scalar curvature in $\mathbb{S}^{n+1}$, and if $n \leq S \leq n+\frac{3}{7} n$, then $S=n$ and $M$ is a minimal Clifford torus. Meanwhile, Wei and Xu [16] proved that if $M$ is a compact minimal hypersurface in $\mathbb{S}^{n+1}$, $n=6,7$, and if $n \leq S \leq n+\tau_{2}(n)$, where $\tau_{2}(n)$ is a positive constant depending only on $n$, then $S \equiv n$ and $M$ is a minimal Clifford torus. Later, Zhang 24] extended the second pinching theorem due to Peng-Terng [12] and Wei-Xu [16] to 8-dimensional compact minimal hypersurfaces in a unit sphere. Recently Ding and Xin [8] obtained the following pinching theorem for $n$-dimensional minimal hypersurfaces in a sphere.

Theorem A. Let $M$ be an n-dimensional compact minimal hypersurface in a unit sphere $\mathbb{S}^{n+1}$, and $S$ the squared length of the second fundamental form of $M$. Then there exists a positive constant $\tau(n)$ depending only on $n$ such that if $n \leq S \leq n+\tau(n)$, then $S \equiv n$, i.e., $M$ is a Clifford torus.

The pinching phenomenon for hypersurfaces of constant mean curvature in spheres is much more complicated than the minimal hypersurface case (see [17], 19]). In [17, Xu proved the following pinching theorem for submanifolds with parallel mean curvature in a sphere.

Theorem B. Let $M$ be an $n$-dimensional compact submanifold with parallel mean curvature vector $(H \neq 0)$ in an $(n+p)$-dimensional unit sphere $\mathbb{S}^{n+p}$. If $S \leq \alpha(n, H)$, then either $M$ is pseudo-umbilical, or $S \equiv \alpha(n, H)$ and $M$ is the isoparametric hypersurface $\mathbb{S}^{n-1}\left(\frac{1}{\sqrt{1+\lambda^{2}}}\right) \times \mathbb{S}^{1}\left(\frac{\lambda}{\sqrt{1+\lambda^{2}}}\right)$ in a great sphere $\mathbb{S}^{n+1}$. In particular, if $M$ is a compact hypersurface with constant mean curvature $H(\neq 0)$ in $\mathbb{S}^{n+1}$, then $M$ is either a totally umbilical sphere $\mathbb{S}^{n}\left(\frac{1}{\sqrt{1+H^{2}}}\right)$, or a Clifford hypersurface $\mathbb{S}^{n-1}\left(\frac{1}{\sqrt{1+\lambda^{2}}}\right) \times \mathbb{S}^{1}\left(\frac{\lambda}{\sqrt{1+\lambda^{2}}}\right)$. Here $\alpha(n, H)=n+\frac{n^{3} H^{2}}{2(n-1)}-\frac{n(n-2)|H|}{2(n-1)} \sqrt{n^{2} H^{2}+4(n-1)}$ and $\lambda=\frac{n|H|+\sqrt{n^{2} H^{2}+4(n-1)}}{2(n-1)}$.

In [20], Xu and Tian generalized Suh-Yang's pinching theorem [15] to the case where $M$ is a compact hypersurface with constant scalar curvature and small constant mean curvature in $\mathbb{S}^{n+1}$. The following second pinching theorem for hypersurfaces with small constant mean curvature was proved for $n \leq 7$ by Cheng-He-Li [3] and Xu-Zhao [21] respectively, and for $n=8$ by $\mathrm{Xu}[22]$.

Theorem C. Let $M$ be an n-dimensional compact hypersurface with constant mean curvature $H(\neq 0)$ in a unit sphere $\mathbb{S}^{n+1}, n \leq 8$. There exist two positive constants $\gamma_{0}(n)$ and $\delta_{0}(n)$ depending only on $n$ such that if $|H| \leq \gamma_{0}(n)$, and $\beta(n, H) \leq S<\beta(n, H)+\delta_{0}(n)$, then $S \equiv \beta(n, H)$ and $M=\mathbb{S}^{1}\left(\frac{1}{\sqrt{1+\mu^{2}}}\right) \times \mathbb{S}^{n-1}\left(\frac{\mu}{\sqrt{1+\mu^{2}}}\right)$. Here $\beta(n, H)=n+\frac{n^{3}}{2(n-1)} H^{2}+$ 
$\frac{n(n-2)}{2(n-1)} \sqrt{n^{2} H^{4}+4(n-1) H^{2}}$ and $\mu=\frac{n|H|+\sqrt{n^{2} H^{2}+4(n-1)}}{2}$.

In this paper, we prove the second pinching theorem for $n$-dimensional hypersurfaces with constant mean curvature, which is a generalization of Theorems A and C.

Main Theorem. Let $M$ be an $n$-dimensional compact hypersurface with constant mean curvature $H$ in a unit sphere $\mathbb{S}^{n+1}$. There exist two positive constants $\gamma(n)$ and $\delta(n)$ depending only on $n$ such that if $|H| \leq \gamma(n)$, and $\beta(n, H) \leq S \leq \beta(n, H)+\delta(n)$, then $S \equiv \beta(n, H)$

and $M$ is one of the following cases: (i) $\mathbb{S}^{k}\left(\sqrt{\frac{k}{n}}\right) \times \mathbb{S}^{n-k}\left(\sqrt{\frac{n-k}{n}}\right), 1 \leq k \leq n-1$; (ii) $\mathbb{S}^{1}\left(\frac{1}{\sqrt{1+\mu^{2}}}\right) \times \mathbb{S}^{n-1}\left(\frac{\mu}{\sqrt{1+\mu^{2}}}\right)$. Here $\beta(n, H)=n+\frac{n^{3}}{2(n-1)} H^{2}+\frac{n(n-2)}{2(n-1)} \sqrt{n^{2} H^{4}+4(n-1) H^{2}}$ and $\mu=\frac{n|H|+\sqrt{n^{2} H^{2}+4(n-1)}}{2}$.

\section{Preliminaries}

Let $M^{n}$ be an $n$-dimensional compact hypersurface with constant mean curvature in a unit sphere $\mathbb{S}^{n+1}$. We shall make use of the following convention on the range of indices.

$$
1 \leq A, B, C, \ldots, \leq n+1, \quad 1 \leq i, j, k, \ldots, \leq n .
$$

For an arbitrary fixed point $x \in M \subset \mathbb{S}^{n+1}$, we choose an orthonormal local frame field $\left\{e_{A}\right\}$ in $\mathbb{S}^{n+1}$ such that $e_{i}$ 's are tangent to $M$. Let $\left\{\omega_{A}\right\}$ be the dual frame fields of $\left\{e_{A}\right\}$ and $\left\{\omega_{A B}\right\}$ the connection 1 -forms of $\mathbb{S}^{n+1}$. Restricting to $M$, we have

$$
\omega_{n+1 i}=\sum_{j} h_{i j} \omega_{j}, h_{i j}=h_{j i}
$$

Let $h$ be the second fundamental form of $M$. Denote by $R, H$ and $S$ the scalar curvature, mean curvature and squared length of the second fundamental form of $M$, respectively. Then we have

$$
\begin{gathered}
h=\sum_{i, j} h_{i j} \omega_{i} \otimes \omega_{j}, \\
S=\sum_{i, j} h_{i j}^{2}, H=\frac{1}{n} \sum_{i} h_{i i}, \\
R=n(n-1)+n^{2} H^{2}-S .
\end{gathered}
$$

We choose $e_{n+1}$ such that $H=\frac{1}{n} \sum_{i} h_{i i} \geq 0$. Denote by $h_{i j k}, h_{i j k l}$ and $h_{i j k l m}$ the first, second and third covariant derivatives of the second fundamental tensor $h_{i j}$, respectively. Then we have

$$
\begin{gathered}
\nabla h=\sum_{i, j, k} h_{i j k} \omega_{i} \otimes \omega_{j} \otimes \omega_{k}, h_{i j k}=h_{i k j}, \\
h_{i j k l}=h_{i j l k}+\sum_{m} h_{m j} R_{m i k l}+\sum_{m} h_{i m} R_{m j k l},
\end{gathered}
$$




$$
h_{i j k l m}=h_{i j k m l}+\sum_{r} h_{r j k} R_{r i l m}+\sum_{r} h_{i r k} R_{r j l m}+\sum_{r} h_{i j r} R_{r k l m} .
$$

At each fixed point $x \in M$, we take orthonormal frames $\left\{e_{i}\right\}$ such that $h_{i j}=\lambda_{i} \delta_{i j}$ for all $i, j$. Then $\sum_{i} \lambda_{i}=n H$ and $\sum_{i} \lambda_{i}^{2}=S$. By a direct computation, we have

$$
\begin{gathered}
\frac{1}{2} \Delta S=S(n-S)-n^{2} H^{2}+n H f_{3}+|\nabla h|^{2}, \\
\frac{1}{2} \Delta|\nabla h|^{2}=(2 n+3-S)|\nabla h|^{2}-\frac{3}{2}|\nabla S|^{2}+\left|\nabla^{2} h\right|^{2} \\
+\sum_{i, j, k, l, m}\left(6 h_{i j k} h_{i l m} h_{j l} h_{k m}-3 h_{i j k} h_{i j l} h_{k m} h_{m l}\right)+3 n H \sum_{i, j, k, l} h_{i j k} h_{j l k} h_{l i} \\
=(2 n+3-S)|\nabla h|^{2}-\frac{3}{2}|\nabla S|^{2}+\left|\nabla^{2} h\right|^{2}+3(2 B-A)+3 n H C,
\end{gathered}
$$

where

$$
f_{k}=\sum_{i} \lambda_{i}^{k}, A=\sum_{i, j, k} h_{i j k}^{2} \lambda_{i}^{2}, B=\sum_{i, j, k} h_{i j k}^{2} \lambda_{i} \lambda_{j}, C=\sum_{i, j, k} h_{i j k}^{2} \lambda_{i} .
$$

Using a similar method as in [11], we obtain

$$
\begin{gathered}
h_{i j i j}=h_{j i j i}+t_{i j}, \\
\left|\nabla^{2} h\right|^{2} \geq \frac{3}{4} \sum_{i \neq j} t_{i j}^{2}=\frac{3}{4} \sum_{i, j} t_{i j}^{2},
\end{gathered}
$$

and

$$
3(A-2 B) \leq a S|\nabla h|^{2}
$$

where $t_{i j}=\left(\lambda_{i}-\lambda_{j}\right)\left(1+\lambda_{i} \lambda_{j}\right)$ and $a=\frac{\sqrt{17}+1}{2}$. From (11), we have

$$
\left|\nabla^{2} h\right|^{2} \geq \frac{3}{2}\left[S f_{4}-f_{3}^{2}-S^{2}-S(S-n)-n^{2} H^{2}+2 n H f_{3}\right]
$$

By a computation, we obtain

$$
\begin{aligned}
\frac{1}{3} \sum_{i, j} h_{i j}\left(f_{3}\right)_{i j} & =\frac{1}{3} \sum_{k} \lambda_{k}\left(f_{3}\right)_{k k} \\
& =\sum_{k} \lambda_{k}\left(\sum_{i} h_{i i k k} \lambda_{i}^{2}+2 \sum_{i, j} h_{i j k}^{2} \lambda_{i}\right) \\
& =\sum_{i, k} h_{i i k k} \lambda_{k} \lambda_{i}^{2}+2 \sum_{i, j, k} h_{i j k}^{2} \lambda_{i} \lambda_{k} \\
& =\sum_{i, k}\left[h_{k k i i}+\left(\lambda_{i}-\lambda_{k}\right)\left(1+\lambda_{i} \lambda_{k}\right)\right] \lambda_{k} \lambda_{i}^{2}+2 B \\
& =\sum_{i}\left(\frac{S_{i i}}{2}-\sum_{j, k} h_{i j k}^{2}\right) \lambda_{i}^{2}+\sum_{i, k} \lambda_{i}^{2} \lambda_{k}\left(\lambda_{i}-\lambda_{k}\right)\left(1+\lambda_{i} \lambda_{k}\right)+2 B \\
& =\sum_{i, j, k} \frac{h_{i k} h_{k j}}{2} S_{i j}+n H f_{3}-S^{2}-f_{3}^{2}+S f_{4}-(A-2 B)
\end{aligned}
$$


Since $\int_{M} \sum_{i, j} h_{i j}\left(f_{3}\right)_{i j} d M=0$, we drive the following integral formula.

$$
\begin{aligned}
\int_{M}(A-2 B) d M & =\int_{M}\left(n H f_{3}-S^{2}-f_{3}^{2}+S f_{4}+\sum_{i, j, k} \frac{h_{i k} h_{k j}}{2} S_{i j}\right) d M \\
& =\int_{M}\left(n H f_{3}-S^{2}-f_{3}^{2}+S f_{4}-\sum_{i, j, k}\left(h_{i k} h_{k j}\right)_{j} \frac{S_{i}}{2}\right) d M \\
& =\int_{M}\left(n H f_{3}-S^{2}-f_{3}^{2}+S f_{4}-\sum_{i, j, k} h_{i k j} h_{k j} \frac{S_{i}}{2}-\sum_{i, j, k} h_{i k} h_{k j j} \frac{S_{i}}{2}\right) d M \\
& =\int_{M}\left(n H f_{3}-S^{2}-f_{3}^{2}+S f_{4}-\sum_{i, j, k} h_{i k j} h_{k j} \frac{S_{i}}{2}\right) d M \\
& =\int_{M}\left(n H f_{3}-S^{2}-f_{3}^{2}+S f_{4}-\frac{|\nabla S|^{2}}{4}\right) d M .
\end{aligned}
$$

\section{Proof of Main Theorem}

The key to the proof of Main Theorem is to establish some integral equalities and inequalities on the second fundamental form of $M$ and its covariant derivatives by the parameter method.

To simplify the computation, we introduce the tracefree second fundamental form $\phi=$ $\sum_{i, j} \phi_{i j} \omega_{i} \otimes \omega_{j}$, where $\phi_{i j}=h_{i j}-H \delta_{i j}$. If $h_{i j}=\lambda_{i} \delta_{i j}$, then $\phi_{i j}=\mu_{i} \delta_{i j}$, where $\mu_{i}=\lambda_{i}-H$.

Putting $\Phi=|\phi|^{2}$ and $\bar{f}_{k}=\sum_{i} \mu_{i}^{k}$, we get $\Phi=S-n H^{2}, f_{3}=\bar{f}_{3}+3 H \Phi+n H^{3}$ and $f_{4}=\bar{f}_{4}+4 H \bar{f}_{3}+6 H^{2} \Phi+n H^{4}$. From (8), we obtain

$$
\begin{aligned}
\frac{1}{2} \Delta \Phi & =S(n-S)-n^{2} H^{2}+n H f_{3}+|\nabla h|^{2} \\
& =-\Phi^{2}+n \Phi+n H \bar{f}_{3}+n H^{2} \Phi+|\nabla \phi|^{2} \\
& =-F(\Phi)+|\nabla \phi|^{2},
\end{aligned}
$$

where $F(\Phi)=\Phi^{2}-n \Phi-n H^{2} \Phi-n H \bar{f}_{3}$. Therefore, we have

$$
|\nabla \Phi|^{2}=\frac{1}{2} \Delta(\Phi)^{2}-\Phi \Delta \Phi=\frac{1}{2} \Delta(\Phi)^{2}+2 \Phi F(\Phi)-2 \Phi|\nabla \phi|^{2},
$$

and

$$
\int_{M} F(\Phi) d M=\int_{M}|\nabla \phi|^{2} d M
$$

Lemma 1.(See [17]) Let $a_{1}, a_{2}, \ldots, a_{n}$ be real numbers satisfying $\sum_{i} a_{i}=0$ and $\sum_{i} a_{i}^{2}=a$. Then

$$
\left|\sum_{i} a_{i}^{3}\right| \leq \frac{n-2}{\sqrt{n(n-1)}} a^{\frac{3}{2}}
$$

and the equality holds if and only if at least $n-1$ numbers of $a_{i}$ 's are same with each other. 
From Lemma 1, we get

$$
\begin{aligned}
F(\Phi) & \geq \Phi^{2}-n \Phi-n H^{2} \Phi-\frac{n(n-2) H \Phi^{\frac{3}{2}}}{\sqrt{n(n-1)}} \\
& =\Phi\left[\Phi-\frac{n(n-2) H \Phi^{\frac{1}{2}}}{\sqrt{n(n-1)}}-n\left(1+H^{2}\right)\right] \\
& \geq 0,
\end{aligned}
$$

provided

$$
\Phi \geq \beta_{0}(n, H):=n+\frac{n^{3}}{2(n-1)} H^{2}+\frac{n(n-2)}{2(n-1)} \sqrt{n^{2} H^{4}+4(n-1) H^{2}}-n H^{2} .
$$

Moreover, $F(\Phi)=0$ if and only if $\Phi=\beta_{0}(n, H)$.

Set

$$
G=\sum_{i, j}\left(\lambda_{i}-\lambda_{j}\right)^{2}\left(1+\lambda_{i} \lambda_{j}\right)^{2}
$$

Then we have

$$
G=2\left[S f_{4}-f_{3}^{2}-S^{2}-S(S-n)+2 n H f_{3}-n^{2} H^{2}\right] .
$$

This together with (8) and (15) implies

$$
\frac{1}{2} \int_{M} G d M=\int_{M}\left[(A-2 B)-|\nabla h|^{2}+\frac{1}{4}|\nabla S|^{2}\right] d M .
$$

Lemma 2. Let $M$ be an $n(\geq 4)$-dimensional compact hypersurface with constant mean curvature in $\mathbb{S}^{n+1}$. If $S \geq \beta(n, H)$, then we have

$$
3(A-2 B) \leq 2 S|\nabla h|^{2}+C_{1}(n)|\nabla h|^{2} G^{\frac{1}{3}},
$$

where $C_{1}(n)=(\sqrt{17}-3)[6(\sqrt{17}+1)]^{-\frac{1}{3}}\left(\frac{2}{\sqrt{17}}-\frac{\sqrt{2}}{17}-\frac{1}{n}\right)^{-\frac{2}{3}}$.

Proof. We derive the estimate above at each fixed point $x \in M$. If $\lambda_{j}^{2}-4 \lambda_{i} \lambda_{j} \leq 2 S$ for all $i \neq j$, then we get the desired estimate immediately. Otherwise, we assume that there exist $i \neq j$, such that $\lambda_{j}^{2}-4 \lambda_{i} \lambda_{j}=t S>2 S$.

We get

$$
S \geq \lambda_{i}^{2}+\lambda_{j}^{2}=\left(\frac{t S-\lambda_{j}^{2}}{4 \lambda_{j}}\right)^{2}+\lambda_{j}^{2}
$$

Then

$$
\lambda_{j}^{2} \leq \frac{1}{17}\left(t+8+4 \sqrt{4+t-t^{2}}\right) S, \quad 2<t \leq \frac{\sqrt{17}+1}{2},
$$

which implies

$$
-\lambda_{i} \lambda_{j} \geq \frac{1}{17}\left(4 t-2-\sqrt{4+t-t^{2}}\right) S \geq 0.26 S>\frac{S}{n} \geq 1 .
$$

On the other hand, we have

$$
\left(\lambda_{i}-\lambda_{j}\right)^{2}=\left(\frac{\lambda_{j}}{2}+\lambda_{i}\right)^{2}+\frac{3}{4}\left(\lambda_{j}^{2}-4 \lambda_{i} \lambda_{j}\right) \geq \frac{3 t}{4} S .
$$


By the definition of $G$, we get

$$
\begin{aligned}
G & \geq 2\left(\lambda_{i}-\lambda_{j}\right)^{2}\left(1+\lambda_{i} \lambda_{j}\right)^{2} \\
& \geq \frac{3 t}{2} S\left(1+\lambda_{i} \lambda_{j}\right)^{2} \\
& \geq \frac{3 t}{2} S\left(-\lambda_{i} \lambda_{j}-\frac{S}{n}\right)^{2} \\
& \geq \frac{3 t}{2}\left[\frac{1}{17}\left(4 t-2-\sqrt{4+t-t^{2}}\right)-\frac{1}{n}\right]^{2} S^{3} .
\end{aligned}
$$

We define an auxiliary function

$$
\zeta(t)=\frac{t}{(t-2)^{3}}\left[\frac{1}{17}\left(4 t-2-\sqrt{4+t-t^{2}}\right)-\frac{1}{n}\right]^{2}, \quad 2<t \leq \frac{\sqrt{17}+1}{2} .
$$

Then we have

$$
\begin{aligned}
\zeta(t) & \geq \frac{t}{(t-2)^{3}}\left[\frac{1}{17}(4 t-2-\sqrt{2})-\frac{1}{n}\right]^{2} \\
& \geq \inf _{2<t \leq \frac{\sqrt{17}+1}{2}} \frac{t}{(t-2)^{3}}\left[\frac{1}{17}(4 t-2-\sqrt{2})-\frac{1}{n}\right]^{2} \\
& =\frac{4(\sqrt{17}+1)}{(\sqrt{17}-3)^{3}}\left(\frac{2}{\sqrt{17}}-\frac{\sqrt{2}}{17}-\frac{1}{n}\right)^{2} .
\end{aligned}
$$

Hence

$$
\begin{aligned}
\left(\lambda_{j}^{2}-4 \lambda_{i} \lambda_{j}-2 S\right)^{3} & =(t-2)^{3} S^{3} \\
& \leq \frac{2 G}{3 \zeta(t)} \\
& \leq \frac{(\sqrt{17}-3)^{3}}{6(\sqrt{17}+1)}\left(\frac{2}{\sqrt{17}}-\frac{\sqrt{2}}{17}-\frac{1}{n}\right)^{-2} G \\
& =\left(C_{1}(n) G^{\frac{1}{3}}\right)^{3} .
\end{aligned}
$$

This implies

$$
\begin{aligned}
3(A-2 B) & \leq \sum_{i, j, k \text { distinct }}\left[2\left(\lambda_{i}^{2}+\lambda_{j}^{2}+\lambda_{k}^{2}\right)-\left(\lambda_{i}+\lambda_{j}+\lambda_{k}\right)^{2}\right] h_{i j k}^{2}+3 \sum_{i \neq j}\left(\lambda_{j}^{2}-4 \lambda_{i} \lambda_{j}\right) h_{i i j}^{2} \\
& \leq 2 S \sum_{i, j, k \text { distinct }} h_{i j k}^{2}+3 \sum_{i \neq j} h_{i i j}^{2}\left(2 S+C_{1}(n) G^{\frac{1}{3}}\right) \\
& \leq 2 S|\nabla h|^{2}+C_{1}(n)|\nabla h|^{2} G^{\frac{1}{3}} .
\end{aligned}
$$

Proof of Main Theorem.(i) When $H=0$, the assertion follows from Theorem A.

(ii) When $H \neq 0$, the assertion for lower dimensional cases $(n \leq 8)$ was verified in [3], 21] 
and [22]. We consider the case for $n \geq 4$. From (10) and (11), we see that $G=\sum_{i, j} t_{i j}^{2}$ and $\left|\nabla^{2} h\right|^{2} \geq \frac{3}{4} G$. Let $0<\theta<1$, we have

$$
\int_{M}\left|\nabla^{2} h\right|^{2} d M \geq\left[\frac{3(1-\theta)}{4}+\frac{3 \theta}{4}\right] \int_{M} G d M
$$

From (9), (21), Lemma 2 and Young's inequality, we drive the following inequality.

$$
\begin{aligned}
\frac{3(1-\theta)}{4} \int_{M} G d M \leq & \int_{M}\left[(S-2 n-3)|\nabla h|^{2}+\frac{3}{2}|\nabla S|^{2}+3(A-2 B)-3 n H C-\frac{3 \theta}{4} G\right] d M \\
= & \int_{M}\left(S-2 n-3+\frac{3 \theta}{2}\right)|\nabla h|^{2} d M+\left(3-\frac{3 \theta}{2}\right) \int_{M}(A-2 B) d M \\
& +\left(\frac{3}{2}-\frac{3 \theta}{8}\right) \int_{M}|\nabla S|^{2} d M-3 n H \int_{M} C d M \\
\leq & \int_{M}\left(S-2 n-3+\frac{3 \theta}{2}\right)|\nabla h|^{2} d M+\left(1-\frac{\theta}{2}\right) \int_{M}\left(2 S|\nabla h|^{2}\right. \\
& \left.+C_{1}(n)|\nabla h|^{2} G^{\frac{1}{3}}\right) d M+\left(\frac{3}{2}-\frac{3 \theta}{8}\right) \int_{M}|\nabla S|^{2} d M-3 n H \int_{M} C d M \\
\leq & \int_{M}\left[(3-\theta) S-2 n-3+\frac{3 \theta}{2}\right]|\nabla h|^{2} d M+\frac{3(1-\theta)}{4} \int_{M} G d M \\
& +C_{2}(n, \theta) \int_{M}|\nabla h|^{3} d M+\left(\frac{3}{2}-\frac{3 \theta}{8}\right) \int_{M}|\nabla S|^{2} d M \\
& -3 n H \int_{M} C d M,
\end{aligned}
$$

where $C_{2}(n, \theta)=\frac{4}{9} C_{1}(n)^{\frac{3}{2}}\left(1-\frac{\theta}{2}\right)^{\frac{3}{2}}(1-\theta)^{-\frac{1}{2}}$.

Let $\epsilon>0$, from (16), we get

$$
\begin{aligned}
\int_{M}|\nabla h|^{3} d M & =\int_{M}|\nabla \phi|^{3} d M \\
& =\int_{M}|\nabla \phi|\left(F(\Phi)+\frac{1}{2} \Delta \Phi\right) d M \\
& =\int_{M} F(\Phi)|\nabla \phi| d M-\frac{1}{2} \int_{M} \nabla|\nabla \phi| \cdot \nabla \Phi d M \\
& \leq \int_{M} F(\Phi)|\nabla \phi| d M+\epsilon \int_{M}\left|\nabla^{2} \phi\right|^{2} d M+\frac{1}{16 \epsilon} \int_{M}|\nabla \Phi|^{2} d M
\end{aligned}
$$

Since

$$
|C| \leq \sqrt{S}|\nabla h|^{2}
$$

we have

$$
\begin{aligned}
0 \leq & \int_{M}\left[(3+3 \sqrt{n} H-\theta)\left(\Phi+n H^{2}\right)-2 n-3+\frac{3 \theta}{2}\right]|\nabla \phi|^{2} d M \\
& +C_{2}(n, \theta)\left[\int_{M} F(\Phi)|\nabla \phi| d M+\epsilon \int_{M}\left|\nabla^{2} \phi\right|^{2} d M+\frac{1}{16 \epsilon} \int_{M}|\nabla \Phi|^{2} d M\right] \\
& +\left(\frac{3}{2}-\frac{3 \theta}{8}\right) \int_{M}|\nabla \Phi|^{2} d M
\end{aligned}
$$


Substituting (12) and (33) into (9), we have

$$
\begin{aligned}
\int_{M}\left|\nabla^{2} \phi\right|^{2} d M & =\int_{M}\left|\nabla^{2} h\right|^{2} d M \\
& \leq \int_{M}\left[(S-2 n-3)|\nabla h|^{2}+\frac{3}{2}|\nabla S|^{2}+a S|\nabla h|^{2}-3 n H C\right] d M \\
& \leq \int_{M}[(a+1+3 \sqrt{n} H) S-2 n-3]|\nabla \phi|^{2} d M+\frac{3}{2} \int_{M}|\nabla S|^{2} d M .
\end{aligned}
$$

Combining (16) and (17), we have

$$
\begin{aligned}
\int_{M} \frac{1}{2}|\nabla \Phi|^{2} d M= & \int_{M} \Phi F(\Phi) d M-\int_{M} \Phi|\nabla \phi|^{2} d M+\beta_{0}(n, H) \int_{M}|\nabla \phi|^{2} d M \\
& -\beta_{0}(n, H) \int_{M} F(\Phi) d M \\
= & \int_{M}\left(\Phi-\beta_{0}(n, H)\right) F(\Phi) d M+\int_{M}\left(\beta_{0}(n, H)-\Phi\right)|\nabla \phi|^{2} d M .
\end{aligned}
$$

Hence

$$
\begin{aligned}
0 \leq & \int_{M}\left\{\left[3+3 \sqrt{n} H-\theta+\epsilon C_{2}(n, \theta)(a+1+3 \sqrt{n} H)\right]\left(\Phi-\beta_{0}(n, H)\right)\right. \\
& +\beta(n, H)\left[3+3 \sqrt{n} H-\theta+\epsilon C_{2}(n, \theta)(a+1+3 \sqrt{n} H)\right] \\
& -2\left(\frac{3}{2}-\frac{3 \theta}{8}+\frac{C_{2}(n, \theta)}{16 \epsilon}+\frac{3 \epsilon C_{2}(n, \theta)}{2}\right)\left(\Phi-\beta_{0}(n, H)\right) \\
& \left.-2 n-3+\frac{3 \theta}{2}-\epsilon C_{2}(n, \theta)(2 n+3)\right\}|\nabla \phi|^{2} d M \\
& +2\left(\frac{3}{2}-\frac{3 \theta}{8}+\frac{C_{2}(n, \theta)}{16 \epsilon}+\frac{3 \epsilon C_{2}(n, \theta)}{2}\right) \int_{M}\left(\Phi-\beta_{0}(n, H)\right) F(\Phi) d M \\
& +C_{2}(n, \theta) \int_{M} F(\Phi)|\nabla \phi| d M \\
= & \int_{M}\left\{D(n, H)\left[3+3 \sqrt{n} H-\theta+\epsilon C_{2}(n, \theta)(a+1+3 \sqrt{n} H)\right]\right. \\
& \left.+(1-\theta) n-3+\frac{3 \theta}{2}+3 n^{\frac{3}{2}} H+\epsilon C_{2}(n, \theta)\left(a n+3 n^{\frac{3}{2}} H-n-3\right)\right\}|\nabla \phi|^{2} d M \\
& -\left(\frac{\theta}{4}+\frac{C_{2}(n, \theta)}{8 \epsilon}-3 \sqrt{n} H+\epsilon C_{2}(n, \theta)(2-a-3 \sqrt{n} H)\right) \int_{M}\left(\Phi-\beta_{0}(n, H)\right)|\nabla \phi|^{2} d M \\
& +\left(3-\frac{3 \theta}{4}+\frac{C_{2}(n, \theta)}{8 \epsilon}+3 \epsilon C_{2}(n, \theta)\right) \int_{M}\left(\Phi-\beta_{0}(n, H)\right) F(\Phi) d M \\
& +C_{2}(n, \theta) \int_{M} F(\Phi)|\nabla \phi| d M,
\end{aligned}
$$

where $\beta(n, H)=\beta_{0}(n, H)+n H^{2}$ and $D(n, H)=\beta(n, H)-n$.

Note that

$$
\frac{\theta}{4}+\frac{C_{2}(n, \theta)}{8 \epsilon}-3 \sqrt{n} H+\epsilon C_{2}(n, \theta)(2-a-3 \sqrt{n} H) \geq 0
$$


for all $\epsilon \in\left(0, \epsilon_{1}\right]$, where $\epsilon_{1}$ is some positive constant. When $\beta(n, H) \leq S \leq \beta(n, H)+\epsilon^{2}$, we obtain

$$
\begin{aligned}
0 \leq & \int_{M}\left[(1-\theta) n-3+\frac{3 \theta}{2}+3 n^{\frac{3}{2}} H+D(n, H)(3+3 \sqrt{n} H-\theta)+O(\epsilon, \theta, H)\right]|\nabla \phi|^{2} d M \\
& +C_{2}(n, \theta) \int_{M} F(\Phi)|\nabla \phi| d M
\end{aligned}
$$

where

$$
\begin{aligned}
O(\epsilon, \theta, H)= & \epsilon D(n, H) C_{2}(n, \theta)(a+1+3 \sqrt{n} H)+\epsilon C_{2}(n, \theta)\left(a n+3 n^{\frac{3}{2}} H-n-3\right) \\
& +\epsilon^{2}\left(3-\frac{3 \theta}{4}+\frac{C_{2}(n, \theta)}{8 \epsilon}+3 \epsilon C_{2}(n, \theta)\right) .
\end{aligned}
$$

On the other hand, we have

$$
C_{2}(n, \theta) \int_{M} F(\Phi)|\nabla \phi| d M \leq \frac{3}{8} \int_{M} F(\Phi) d M+\frac{2 C_{2}(n, \theta)^{2}}{3} \int_{M} F(\Phi)|\nabla \phi|^{2} d M .
$$

Using Lemma 1, we drive an upper bound for $F(\Phi)$.

$$
\begin{aligned}
F(\Phi) & \leq \Phi^{2}-n \Phi-n H^{2} \Phi+\frac{n(n-2) H \Phi^{\frac{3}{2}}}{\sqrt{n(n-1)}} \\
& =\Phi\left[\Phi+\frac{n(n-2) H \Phi^{\frac{1}{2}}}{\sqrt{n(n-1)}}-n\left(1+H^{2}\right)\right] \\
& =\frac{\Phi\left(\Phi^{\frac{1}{2}}+\beta_{0}(n, H)^{\frac{1}{2}}\right)\left(\Phi-\alpha_{0}(n, H)\right)}{\Phi^{\frac{1}{2}}+\alpha_{0}(n, H)^{\frac{1}{2}}}
\end{aligned}
$$

where $\alpha_{0}(n, H)=\left[\frac{-n(n-2) H+n \sqrt{n^{2} H^{2}+4 n-4}}{2 \sqrt{n(n-1)}}\right]^{2}$.

When $\delta(n) \leq \epsilon^{2}$ and $\epsilon \leq 1$, we choose positive constant $\gamma_{1}(n)$ such that $n \leq \Phi \leq 2 n$ and $x_{1} \leq 2 \sqrt{n}$ for all $H \leq \gamma_{1}(n)$. We obtain

$$
F(\Phi) \leq 8 n\left(\Phi-\alpha_{0}(n, H)\right) \leq 8 n\left(\epsilon^{2}+\frac{n(n-2)}{(n-1)} \sqrt{n^{2} H^{4}+4(n-1) H^{2}}\right) .
$$

Let $\theta=\theta(n)=1-\frac{1}{8 n}$. We choose positive constants $\gamma_{2}(n)$ and $\gamma_{3}(n)$ such that $3 n^{\frac{3}{2}} H+$ $D(n, H)(3+3 \sqrt{n} H) \leq \frac{1}{8}$ for all $H \leq \gamma_{2}(n)$, and $\frac{16 n^{2}(n-2)}{(n-1)} \sqrt{n^{2} \gamma_{3}(n)^{4}+4(n-1) \gamma_{3}(n)^{2}} \leq$ $\frac{9}{16 C_{2}(n, \theta(n))^{2}}$.

Take $\epsilon_{2}(n)=\left[\frac{n(n-2)}{(n-1)} \sqrt{n^{2} \gamma_{3}(n)^{4}+4(n-1) \gamma_{3}(n)^{2}}\right]^{\frac{1}{2}}>0$. Combining (39), (40) and (42), we obtain

$$
\int_{M}\left[-\frac{1}{2}+O(\epsilon, \theta(n), H)\right]|\nabla \phi|^{2} d M \geq 0
$$

for all $H \leq \gamma(n)=\min \left\{\gamma_{1}(n), \gamma_{2}(n), \gamma_{3}(n)\right\}$ and $\epsilon \leq \min \left\{\epsilon_{1}, \epsilon_{2}(n)\right\}$.

For $\epsilon \leq 1$, we have

$$
O(\epsilon, \theta(n), H) \leq \epsilon D(n, \gamma(n)) C_{2}(n, \theta(n))(a+1+3 \sqrt{n} \gamma(n))
$$




$$
\begin{aligned}
& +\epsilon C_{2}(n, \theta(n))\left(a n+3 n^{\frac{3}{2}} \gamma(n)\right) \\
& +\epsilon\left(3-\frac{3 \theta(n)}{4}+\frac{C_{2}(n, \theta(n))}{8}+3 C_{2}(n, \theta(n))\right) \\
:= & \epsilon \eta(n),
\end{aligned}
$$

where $a=\frac{\sqrt{17}+1}{2}$.

For $\epsilon \leq \epsilon_{1}(n)$, where $\epsilon_{1}(n)=\frac{C_{2}(n, \theta(n))}{8\left[3 \sqrt{n} \gamma(n)+C_{2}(n, \theta(n))(a+3 \sqrt{n} \gamma(n)-2)\right]}>0, a=\frac{\sqrt{17}+1}{2}$, we have

$$
\frac{C_{2}(n, \theta(n))}{8 \epsilon} \geq 3 \sqrt{n} \gamma(n)+C_{2}(n, \theta(n))(a+3 \sqrt{n} \gamma(n)-2)-\frac{\theta(n)}{4} .
$$

So

$$
\frac{\theta(n)}{4}+\frac{C_{2}(n, \theta(n))}{8 \epsilon}-3 \sqrt{n} H+\epsilon C_{2}(n, \theta(n))(2-a-3 \sqrt{n} H) \geq 0 .
$$

Taking $\delta(n)=\epsilon(n)^{2}$, where $\epsilon(n)=\min \left\{1, \epsilon_{1}(n), \epsilon_{2}(n), \epsilon_{3}(n)\right\}$ and $\epsilon_{3}(n)=\frac{1}{3 \eta(n)}$, we have $\delta(n)>0$. From (43) and the assumption that $\beta(n, H) \leq S \leq \beta(n, H)+\delta(n)$, we obtain $\nabla \phi=0$. This implies $F(\Phi)=0$ and $\Phi=\beta_{0}(n, H)$.

By Lemma 1, we have

$$
\begin{gathered}
\lambda_{1}=\ldots=\lambda_{n-1}=H-\sqrt{\frac{\beta(n, H)-n H^{2}}{n(n-1)}}, \\
\lambda_{n}=H+\sqrt{\frac{(n-1)\left(\beta(n, H)-n H^{2}\right)}{n}} .
\end{gathered}
$$

Therefore $M$ is the Clifford hypersurface

$$
\mathbb{S}^{1}\left(\frac{1}{\sqrt{1+\mu^{2}}}\right) \times \mathbb{S}^{n-1}\left(\frac{\mu}{\sqrt{1+\mu^{2}}}\right)
$$

in $\mathbb{S}^{n+1}$, where $\mu=\frac{n H+\sqrt{n^{2} H^{2}+4(n-1)}}{2}$. This completes the proof of Main Theorem.

Finally we would like to propose the following problems.

Open Problem A. Let $M$ be an n-dimensional compact hypersurface with constant mean curvature $H$ in the unit sphere $\mathbb{S}^{n+1}$. Does there exist a positive constant $\delta(n)$ depending only on $n$ such that if $\beta(n, H) \leq S \leq \beta(n, H)+\delta(n)$, then $S \equiv \beta(n, H)$ ?

Open Problem B. For an n-dimensional compact hypersurface $M^{n}$ with constant mean curvature $H$ in $\mathbb{S}^{n+1}$, set $\mu_{k}=\frac{n|H|+\sqrt{n^{2} H^{2}+4 k(n-k)}}{2 k}$. Suppose that $\alpha(n, H) \leq S \leq \beta(n, H)$. Is it possible to prove that $M$ must be the isoparametric hypersurface $S^{k}\left(\frac{1}{\sqrt{1+\mu_{k}^{2}}}\right) \times S^{n-k}\left(\frac{\mu_{k}}{\sqrt{1+\mu_{k}^{2}}}\right)$, $k=1,2, \cdots, n-1$ ?

When $H=0$, the rigidity theorem due to Lawson [9], Chern, do Carmo and Kobayashi [2] provides an affirmative answer for Open Problem B.

Acknowledgement. We would like to thank Dr. En-Tao Zhao for his helpful discussions. Thanks also to Professor Y. L. Xin for sending us the reference [8]. 


\section{References}

[1] S. P. Chang, On minimal hypersurfaces with constant scalar curvatures in $S^{4}, J$. Differential Geom., 37(1993), 523-534.

[2] S. S. Chern, M. do Carmo, and S. Kobayashi, Minimal submanifolds of a sphere with second fundamental form of constant length, in Functional Analysis and Related Fields, Springer-Verlag, New York, 1970.

[3] Q. M. Cheng, Y. J. He and H. Z. Li, Scalar curvature of hypersurfaces with constant mean curvature in a sphere, Glasgow Math. J., 51(2009), 413-423.

[4] Q. M. Cheng and S. Ishikawa, A characterization of the Clifford torus, Proc. Amer. Math. Soc., 127(1999), 819-828.

[5] Q. M. Cheng and H. C. Yang, Chern's conjecture on minimal hypersurfaces, Math. Z., 227(1998), 377-390.

[6] S. Y. Cheng, On the Chern conjecture for minimal hypersurface with constant scalar curvatures in the spheres, Tsing Hua Lectures on Geometry and Analysis, International Press, Cambridge, MA, 1997, pp. 59-78.

[7] Q. Ding, On spectral characterizations of minimal hypersurfaces in a sphere, Kodai Math. J., 17(1994), 320-328.

[8] Q. Ding and Y. L. Xin, Some results on Chern's problem, preprint, 2010.

[9] B. Lawson, Local rigidity theorems for minimal hypersurfaces, Ann. of Math., 89(1969), 187-197.

[10] A. M. Li and J. M. Li, An intrinsic rigidity theorem for minimal submanifolds in a sphere, Arch. Math., 58(1992), 582-594.

[11] C. K. Peng and C. L. Terng, Minimal hypersurfaces of sphere with constant scalar curvature, Ann. of Math. Study., 103(1983), 177-198.

[12] C. K. Peng and C. L. Terng, The scalar curvature of minimal hypersurfaces in spheres, Math. Ann., 266(1983), 105-113.

[13] J. Simons, Minimal varieties in Riemannian manifolds, Ann. of Math., 88(1968), $62-105$.

[14] S. Y. Cheng and S. T. Yau, Hypersurfaces with constant scalar curvature, Math. Ann., 225(1977), 195-204.

[15] Y. J. Suh. and H. Y. Yang, The scalar curvature of minimal hypersurfaces in a unit sphere, Comm. Contemporary Math., 9(2007), 183-200.

[16] S. M. Wei and H. W. Xu, Scalar curvature of minimal hypersurfaces in a sphere, Math. Res. Lett., 14(2007), 423-432. 
[17] H. W. Xu, A rigidity theorem for submanifolds with parallel mean curvature in a sphere, Arch. Math., 61(1993), 489-496.

[18] H. W. Xu, On closed minimal submanifolds in pinched Riemannian manifolds, Trans. Amer. Math. Soc., 347(1995), 1743-1751.

[19] H. W. Xu, A gap of scalar curvature for higher dimensional hypersurfaces with constant mean curvature, Appl. Math. J. Chinese Univ. Ser. A, 8(1993), 410-419.

[20] H. W. Xu and L. Tian, A new pinching theorem for closed hypersurfaces with constant mean curvature in $S^{n+1}$, preprint, 2009.

[21] H. W. Xu and E. T. Zhao, A characterization of Clifford hypersurface, preprint, 2008 .

[22] Z. Y. Xu, Rigidity theorems for compact minimal hypersurfaces in a sphere, Bachelor Thesis, S.-T. Yau Mathematics Elite Class, Zhejiang University, 2010.

[23] S. T. Yau, Submanifolds with constant mean curvature. I, II, Amer. J. Math., 96, 97(1974, 1975), 346-366, 76-100.

[24] Q. Zhang, The pinching constant of minimal hypersurfaces in the unit spheres. Proc. Amer. Math. Soc., 138(2010), 1833-1841.

Center of Mathematical Sciences

Zhejiang University

Hangzhou 310027

China

e-mail address: xuhw@cms.zju.edu.cn; srxwing@zju.edu.cn 